\title{
Transforming Growth Factor- $\beta 1$ in Congenital Ureteropelvic Junction Obstruction: Diagnosis and Follow-up
}

\author{
Cristian Sager, Juan C. Lopez, Victor Duran, Carol Burek, Ernesto Perazzo \\ Urology Department, Hospital de Pediatria Dr. J. P. Garrahan, Buenos Aires, Argentina
}

\begin{abstract}
Objective: To assess the role of transforming growth factor- $\beta 1$ (TGF- $\beta 1$ ) in congenital ureteropelvic junction obstruction at diagnosis and during postoperative follow-up.

Materials and Methods: We conducted a case-control study including 19 patients with a mean age of 6.7 years and 19 matched controls. All patients presented negative voiding cystourethrography, obstructive diuretic renogram and underwent dismembered pyeloplasty. Urinary TGF- $\beta 1$ and other markers were measured pre-, intra- and postoperatively.

Results: The mean bladder urine TGF- $\beta 1$ concentration in obstructed patients prior to pyeloplasty was higher than in controls $(92.5 \mathrm{pg} / \mathrm{mL} \pm 16.8$ vs. $35.8 \mathrm{pg} / \mathrm{mL} \pm 16.2 ; \mathrm{p}=0.0001)$. The mean renal pelvic urine TGF- $\beta 1$ concentration in the hydronephrotic kidney was higher than in the preoperative bladder urine sample $(122.3 \mathrm{pg} / \mathrm{mL} \pm 43.9 \mathrm{vs} .92 .5 \mathrm{pg} / \mathrm{mL}$ $\pm 16.8 ; \mathrm{p}=0.036)$. Postoperative mean TGF- $\beta 1$ concentration was significantly lower than preoperative TGF- $\beta 1$ (48.7 $\mathrm{pg} / \mathrm{mL} \pm 13.1$ vs. $92.5 \mathrm{pg} / \mathrm{mL} \pm 16.8 ; \mathrm{p}=0.0001)$.

Conclusion: TGF- $\beta 1$ is a cytokine leading to renal fibrosis. The measurement of urinary TGF- $\beta 1$ could become a useful tool for the diagnosis of obstructive hydronephrosis and the evaluation of the parenchyma function status, pre and postoperatively.
\end{abstract}

Key words: kidney pelvis; congenital abnormalities; UPJ; hydronephrosis; transforming growth factor beta-1 Int Braz J Urol. 2009; 35: 315-25

\section{INTRODUCTION}

Since the use of ultrasonography in the follow-up of pregnancies, urologists have been confronted with the progression of fetal and postnatal hydronephrosis. Ureteropelvic junction obstruction (UPJO) has been identified as the most common cause of hydronephrosis in children and its management usually generates controversy (1).

Currently, the criteria for the management of UPJO are based on renal characteristics shown on serial ultrasonography, diuretic renogram, ${ }^{99 \mathrm{~m}} \mathrm{Tc}$ dimercaptosuccinic acid (DMSA) scintigraphy, and rarely, on the excretory urogram. The negative response to a diuretic stimulus and, especially, renal function loss indicate the need for surgical intervention (pyeloplasty). In many cases, the diuretic renogram is the testing modality of choice. Although the test is invasive and uses ionizing irradiation, and even measuring the clearance half-time (T 1/2) is controversial, it is still adopted as the clinical management tool to assist in the evaluation of hydronephrosis $(2,3)$.

Many cases of hydronephrosis may resolve spontaneously; others may result in the deterioration of the parenchymal function, and those treated with pyeloplasty continue to present deterioration in renal function after surgery (4). This erratic course of UPJO under follow-up or surgical intervention creates the need to have simple and more accurate tools available for the diagnosis of early renal function deterioration 
due to obstruction before it becomes irreversible (1).

The pathophysiology of obstructive uropathy involves a complex mechanism that causes alteration in the expression of different growth factors (5). Growth factors are involved in the processes of renal wound healing (6). Transforming growth factor- $\beta 1$ (TGF- $\beta 1$ ) is a cytokine detectable in the urine in cases of normal and pathological conditions of the urinary tract, specifically vesicoureteral reflux and hydronephrosis (7-10).

The aim of the present study was to assess the role of TGF- $\beta 1$ in congenital ureteropelvic junction obstruction at diagnosis and after pyeloplasty. Furthermore, we also correlated this cytokine with other traditional urinary biomarkers and renal scintigraphy.

\section{MATERIALS AND METHODS}

We conducted a prospective case-control study, with surgical intervention performed by a single surgeon, including pediatric outpatients who were treated at the Urology Department in Hospital de Pediatria - Dr. Juan P. Garrahan, from January 2005 to January 2006.

A total of 28 patients were assessed in the present study. Nine were excluded, since they failed to complete the required studies on time. The sample population included 19 patients; 11 boys and 8 girls with a mean age of 6.7 years (SD \pm 5.6 years; range: 5 months to 17.6 years) and 19 matched controls.

We evaluated all consecutive patients of up to 18 years of age, male and female, with diagnosed or suspected unilateral or bilateral UPJO detected on prenatal or postnatal ultrasonography, with normal renal function, under antibiotic prophylaxis.

Hydronephrosis was diagnosed antenatally in nine children and the initial presentation of the remaining ten was persistent low back pain in five, a flank mass in four and nausea/vomiting in one.

Imaging test - The inclusion criteria required an ultrasonography with pelvic dilatation $\geq 15 \mathrm{~mm}$ and/or caliectasis, with no visualization of the distal ureter. Hydronephrosis was classified into grades I to
IV according to the Society for Fetal Urology classification (11).

Once hydronephrosis was detected, patients with normal voiding cystourethrography were subjected to a diuretic renogram. For this test, patients had to be normally hydrated and received an extra fluid intake of 7 to $14 \mathrm{~mL} / \mathrm{kg}$ one hour prior to the test. Technetium-99m-labeled diethylenetriamine pentaacetic acid (99mTc-DTPA) was administered intravenously (200 MBq, minimum dose $20 \mathrm{MBq}$ ). Twenty minutes after the radiotracer injection, furosemide was injected intravenously at a dose of $1 \mathrm{mg} / \mathrm{kg}$ of body weight. Clearance half-time of the tracer (T $1 / 2$ ) from the collecting system was then measured. A $\mathrm{T} 1 / 2 \geq 20$ minutes with increased activity in the collecting system or delayed drainage was identified as obstructive. A T $1 / 2<10$ minutes with a quick washout on the images was defined as non-obstructive and the patient was excluded from the present protocol. A $\mathrm{T} 1 / 2$ between 10 and 20 minutes was considered an equivocal result. In this third case, patients underwent a diuretic excretory urogram and were reassigned to one of the other two groups according to the results.

Renal scintigraphy was performed using an intravenous $99 \mathrm{mTc}-\mathrm{DMSA}$ injection at a dose according to patient weight. Twenty-four hours after the injection, the percentage of functioning renal parenchyma was calculated (normal value: $50 \pm 5 \%$ ).

The diuretic excreting urogram (intravenous pyelography) required a state of euhydration. Nonionic, water-soluble, iodine contrast was used at a dose of $1 \mathrm{~mL} / \mathrm{kg}$ of body weight. Twenty-minutes after the intravenous contrast injection, furosemide was administered at $1 \mathrm{mg} / \mathrm{kg}$ of body weight.

The urograms were classified as obstructive or non-obstructive. The obstructive pattern presented radiographic opacification of the renal parenchyma and pyelogram with renal blush still evident two minutes after intravenous injection of the contrast agent. In the affected kidney, the contrast material became visible later than in the healthy kidney. There was dilatation of the renal pelvis and calices with no visualization of the distal ureter. Twenty minutes after diuretic stimulus, there was persistence of the isotope in the affected kidney, low back pain and total washout of the unobstructed renal unit. The non-obstructive pattern included total washout of the contrast agent from the 
upper excretory system. The latter result implied the exclusion of the patient from the present protocol.

The exclusion criteria also included vesicoureteral reflux, duplex excretory system, megaureters, ureteroceles, posterior urethral valves, renal dysplasia, multicystic kidney, horseshoe kidney, ectopic kidney, solitary functioning kidney, kidney disease, kidney failure, present or past urinary infection, previous urinary tract surgeries, and diabetes.

Laboratory studies - Renal function was assessed by urea, serum creatinine, and creatinine clearance calculated as height/creatinine ratio Schwartz formula (12). The evaluation was done with the collaboration of the Nephrology Department. Urine samples were collected by spontaneous voiding after completing the imaging tests (preoperative urine samples) and the following markers were measured:

a) Proteinuria: 24-hour urine samples were collected and measured by the dipstick colorimetric test. Values $>5 \mathrm{mg} / \mathrm{kg} /$ day or $150 \mathrm{mg} /$ day were considered positive (abnormal). In the case of incontinent patients, proteinuria values were measured in a single urine sample and corrected for urine creatinine concentration. Values $>0.2$ were defined as positive (13-15).

b) Microalbuminuria: 12-hour urine samples were collected and measured by the immunoturbidimetric method. Values $>20 \mu / \mathrm{mL}$ were considered positive. In incontinent patients, microalbuminuria was measured in a single urine sample and corrected for urine creatinine values (13-15).

c) $\beta_{2}$-microglobulin $\left(\beta_{2}-\mathrm{MG}\right)$ : Values from $5 \mu \mathrm{g} / \mathrm{L}$ to $150 \mu \mathrm{g} / \mathrm{L}$ or $<0.20 \mathrm{mg} / \mathrm{l}$ were considered within the normal range (16).

d) TGF- $\beta 1$ : urinary TGF- $\beta 1$ concentration was measured using a commercially available, quantitative sandwich enzyme-linked immunoassay (Elisa) kit (Quantikine; R\&D Systems). Standard curves were created and the values obtained were expressed as $\mathrm{pg} / \mathrm{mL}$ (17).

Treatment - The 19 patients with obstructive hydronephrosis on DTPA renogram underwent surgical intervention. Anderson-Hynes dismembered pyeloplasty was the technique used in all cases. After induction of anesthesia and lumbotomy approach, prior to pyeloplasty, urine samples were obtained from the renal pelvis by needle aspiration to calculate $\beta 2$ -
MG and TGF- $\beta 1$ levels (intraoperative urine samples). An intrapelvic rubber drain was left indwelling for 5 days and a perirenal rubber drain was left for 10-12 days in the incision site.

The 19 UPJO biopsies obtained during surgery were analyzed.

Follow-up - It consisted in measuring urinary markers and performing a renal ultrasonography three months after surgery on patients without pig tail stent and nephrostomy tube. In the case of patients with stent pig tail and nephrostomy tube, the evaluation was done five months after surgery. Urine samples were collected by spontaneous voiding to calculate proteinuria; $\beta 2-\mathrm{MG}$ and TGF- $\beta 1$ levels (postoperative urine samples). The renal pelvis diameter was assessed by renal ultrasonography. The percentage of diameter change was calculated in relation to the preoperative diameter of the renal pelvis. When the serial ultrasounds (at least two tests) showed the same renal pelvis diameter or greater than preoperative values, then a diuretic renogram and DMSA scan was performed.

No episodes of urinary infection were observed 3 and 5 months postoperatively. All patients remained asymptomatic during the follow-up period.

Healthy controls - Patients with no renal pathology at the time of the preoperative evaluation were considered healthy controls. The absence of renal pathology was confirmed by laboratory tests (renal function) and a renal ultrasonography was performed to show the presence of both renal masses. Urine samples from controls were collected by spontaneous voiding after completing the imaging tests and analyzed following the same procedures as in the case of the obstructive hydronephrosis group.

Statistical analysis - Data were analyzed with Statistic software package version 7.0. Qualitative variables were expressed as percentages and quantitative variables as mean \pm standard deviation. To compare two quantitative variables with normal distribution of data, a t-test and linear regression analysis were used. $\mathrm{P}<0.05$ (CI 95\%) was considered statistically significant.

The protocol was approved by the Ethics Committee and the Education and Research Department at Hospital Garrahan. An informed consent was obtained from all children's parents or legal guardians. 


\section{RESULTS}

A total of 28 patients were assessed in the present study. Nine were excluded, since they failed to complete the required studies on time. The sample population included 19 patients; 11 boys and 8 girls with a mean age of 6.7 years $(\mathrm{SD} \pm 5.6$ years; range: 5 months to 17.6 years).

Following the classification by the Society for Fetal Urology (11), most patients in the current study presented grade IV hydronephrosis (15 cases, $79 \%)$; 2 cases $(10.5 \%)$ presented grade III; and the remaining 2 cases (10.5\%), grade II. Only one patient presented bilateral hydronephrosis (grade IV on the left and grade I on the right).

Nineteen DTPA renograms were performed and 18 cases revealed typical obstructive curves; only one patient presented an uncertain pattern of curves and required a diuretic excreting urogram, which showed obstructive hydronephrosis. The two patients with grade II hydronephrosis presented renal pelvis anteroposterior diameter $>15 \mathrm{~mm}$ on ultrasound, and obstructed washout curve on diuretic renography.

Nineteen DMSA scintigraphies were performed: 3 renal units presented only scars; 5 units showed less than $40 \%$ of relative renal function; and 3 units had scars and reduced function (less than 40\%). The cause of renal scarring was not identified, despite having excluded patients from our study group that presented or had a history of urinary infection, and those with vesicoureteral reflux (commonly associated to scarring). The remaining eight patients presented preserved renal function in the affected renal unit.

All 19 patients underwent Anderson-Hynes dismembered pyeloplasty, performed by a single surgeon. Subcostal extraperitoneal flank approach with lumbotomy incision was employed. Anastomosis was completed with fine running absorbable sutures. In all patients, a perirenal rubber drain was left for 10-12 days near line of repair. In 16 patients an intrapelvic rubber drain $1 \mathrm{~cm}$ in diameter was used. The proximal diversion was made transversely in the renal pelvis above the suture line. The drain was left for 5 days in the incision site.

Nephrostomy tubes were also placed in two patients, since the renal units presented significant hydronephrosis and reduction in the parenchymal thick- ness. A 9-year-old patient required a second surgical intervention for perirenal hematoma and urinoma. A nephrostomy tube was placed during open surgery.

In two patients, pigtail catheters were used for the ureteropelvic junction, due to suture difficulties. The nephrostomy tubes and the pigtail catheters were removed one month after surgery. In these five patients, urinary laboratory controls were performed five months following surgical intervention.

Obstruction was confirmed by histological findings of the obstructed segment, such as muscle hypertrophy/hyperplasia and collagen deposition, subepithelial fibrosis, and mild inflammatory infiltrate. This typical pattern of UPJO was confirmed in 13 patients. An uncertain UPJO pattern was reported in 6 patients. They presented vascular congestion, edema, mild inflammatory infiltrate with predominance of lymphocytes.

In the postoperative follow-up (three or five months), concomitantly with a significant decrease in urinary TGF- $\beta 1$ values, the renal pelvis decreased on average $56 \%$ in 18 patients, compared with preoperative values. In only one patient, the anteroposterior diameter of the renal pelvis increased by $17 \%$ on three ultrasound examinations, the renogram showed uncertain curves and obstructive pattern was ruled out after the diuretic urogram. This patient was asymptomatic and had normal urine tests results. Renal scintigraphy showed $47 \%$ of relative renal function, the same value as in the preoperative evaluation. For that reason we did not perform diuretic renograms on all patients during postoperative follow-up.

The control group comprised of 19 children: 10 boys and 9 girls with no renal pathology at the time of the preoperative evaluation. The mean age \pm SD was $9.7 \pm 4.4$ years (range: $1.1-5.6$ years). No significant differences were found in the age of both groups (6.7 years \pm 5.6 vs. $9.72 \pm 4.4 ; \mathrm{p}=0.07$ ).

\section{Urinary TGF- $\beta 1$ Concentration}

Mean bladder urine TGF- $\beta 1$ concentration in the obstructed group (preoperative urine sample) was higher than in the control group $(92.5 \mathrm{pg} / \mathrm{mL}$ \pm 16.8 vs. $35.8 \mathrm{pg} / \mathrm{mL} \pm 16.2 ; \mathrm{p}=0.0001)$. Mean renal pelvic urine TGF- $\beta 1$ concentration in patients 
with hydronephrosis (intraoperative urine sample) was higher than in the preoperative bladder urine sample (122.3 $\mathrm{pg} / \mathrm{mL} \pm 43.9$ vs. $92.5 \mathrm{pg} / \mathrm{mL} \pm 16.8 ; \mathrm{p}$ $=0.036)$. Mean bladder urine TGF- $\beta 1$ concentration (postoperative urine sample) was significantly lower than the mean preoperative concentration (48.7 $\mathrm{pg} / \mathrm{mL} \pm 13.1$ vs. $92.5 \mathrm{pg} / \mathrm{mL} \pm 16.8 ; \mathrm{p}=0.0001)$ (Table-1). The levels of TGF- $\beta 1$ were not correlated with creatinine levels, since TGF- $\beta 1$ values in our sample (pre, intra and postoperatively) and in comparison with the controls levels showed statistically significant differences.

In our series, we determined the TGF- $\beta 1$ cut-off point as $39.75 \mathrm{pg} / \mathrm{mL}$, which resulted from the third quartile of the TGF- $\beta 1$ level measured in healthy controls. That means that patients with a TGF- $\beta 1$ level above $39.75 \mathrm{pg} / \mathrm{mL}$ have a 4.25 -fold relative risk (RR) (CI 95\%, 1.08-10.01) of having obstructive hydronephrosis compared with levels below $39.75 \mathrm{pg} / \mathrm{mL}$.

A simple linear regression analysis was used to evaluate the relationship between the percentage of relative renal function loss as measured by DMSA scintigraphy and the intraoperative TGF- $\beta 1$ concentration. We found that for each unit of function loss, TGF- $\beta 1$ increased by $4.54 \mathrm{pg} / \mathrm{mL}$ starting from 79.55 $\mathrm{pg} / \mathrm{mL}$, with a correlation ratio of $0.54(\mathrm{p}=0.03$; statistically significant difference). Therefore, in samples obtained from the affected renal pelvis there was a linear correlation between percentage of relative renal function loss and TGF- $\beta 1$ levels.

In addition, we evaluated whether there was any linear correlation between the chronological age of patients with UPJO and intrapelvic TGF- $\beta 1$ levels. The results showed no relationship between the two variables studied (r 2:0.072; $p=0.39)$.

\section{Proteinuria}

Eighteen patients tested negative for proteinuria. A 3-year-old girl presented $12 \mathrm{mg} / \mathrm{k} /$ day proteinuria (grade IV hydronephrosis; $50 \%$ of relative renal function of the left kidney; preoperative TGF$\beta 1,98 \mathrm{pg} / \mathrm{mL}$; intraoperative TGF- $\beta 1,168 \mathrm{pg} / \mathrm{mL}$, and postoperative TGF- $\beta 1,42 \mathrm{pg} / \mathrm{mL}$ ). Proteinuria values were within the normal range three months after surgery.
Table 1 - Urinary TGF- $\beta 1$ values $(\mathrm{pg} / \mathrm{mL})$.

\begin{tabular}{lcc}
\hline Urine Sample & $\begin{array}{c}\text { Mean Value } \\
(\mathrm{pg} / \mathrm{mL})\end{array}$ & \pm SD \\
\hline Preoperative (vesical) & 92.5 & 16.8 \\
Intraoperative (pelvis) & 122.3 & 43.9 \\
Postoperative (vesical) & 48.7 & 13.1 \\
Controls (vesical) & 35.8 & 16.2 \\
\hline
\end{tabular}

\section{Microalbuminuria}

It was negative in all cases, both preoperatively and postoperatively.

\section{ß2-Microglobulin}

It was negative in 16 cases. Three cases were positive: one in preoperative urine sample (0.6 $\mathrm{mg} / \mathrm{L})$; the remaining two, in intraoperative samples. Of these two, one $(3.47 \mathrm{mg} / \mathrm{L})$ also tested positive for proteinuria. The other patient, the only one with bilateral hydronephrosis, presented $1.4 \mathrm{mg} / \mathrm{L}$. All of them were normalized three months after surgery.

No correlation was found between abnormal values of these last three urinary biomarkers and the grade of hydronephrosis or TGF- $\beta 1$ values.

\section{COMMENTS}

In hydronephrosis, renal ultrasonography has the capacity to define characteristics such as parenchymal appearance, diameter of the renal pelvis and, especially, the calyceal status, all of which determine the grade of hydronephrosis. Once hydronephrosis is diagnosed, no matter how timely medical intervention might be, we believe it is not early enough, since adverse effects in the function of the renal mass can be observed as a consequence of obstruction to the urinary outflow (1).

In our series, most patients presented grade IV hydronephrosis with different ultrasonographic characteristics. This heterogeneity makes this classification of little use in defining the functional status. 
Furthermore, for an accurate diagnosis of hydronephrosis, it is necessary to perform serial ultrasound studies over a period of time and then decide whether further studies are necessary.

On the other hand, the diuretic renogram is currently used and it is preferred over the excretory urography, since it is characterized by reduced radiation and lack of nephrotoxicity. However, the literature shows that diagnosis is uncertain when there is partial excretory response. In addition, several factors influence $\mathrm{T} 1 / 2$, including renal maturity, patient hydration, distensibility and volume of the collecting system, presence of a bladder catheter, among others (18).

In our sample population, we had to perform an excretory urogram on a 10-year-old girl with leftsided grade III hydronephrosis who had undergone DMSA scintigraphy, with 30\% relative renal function, and also had a renogram curve that appeared undifferentiated at phase 2 and 3 . On excretory urography she presented an inadequate response to the diuretic, with typical obstructive pattern. Therefore, we can assume that although the diuretic renogram and DMSA are currently useful tools available for the evaluation of uronephrosis, they cannot be considered ideal.

The inaccuracy of certain complementary studies and the erratic development of UPJO creates the need detect the renal injury at an early stage and to have effective tools for the diagnosis and follow-up of hydronephrosis that can be easy to use, reproducible, reliable, and little or non-invasive, especially in pediatric populations (1). Nevertheless, in the past few years, significant advances have been made in the pathophysiology of obstructive uropathy, with a special emphasis on the pro-inflammatory components.

The obstruction of the upper urinary tract generates a cascade of molecular and histological phenomena $(19,20)$. The renin-angiotensin system is activated, which stimulates the expression of profibrotic cytokines and the transcription factors, including tumor necrosis factor- $\alpha$ (TNF- $\alpha)$, nuclear factor- $\kappa B$ $(\mathrm{NF}-\kappa \mathrm{B})$ and transforming growth factor- $\beta 1$ (TGF- $\beta 1$ ) $(8,20-24)$. Studies have revealed that the protein and gene of TGF- $\beta 1$ are mainly localized at the proximal tubular cells in kidney with hydronephrosis. It has been suggested that the epithelial-to-mesenchymal transition is a major factor leading to renal fibrosis in congenital hydronephrosis (25).

TGF- $\beta 1$ is a $25 \mathrm{Kda}$ cytokine composed of two subunits connected by disulfide bond. This protein is synthesized as a prohormone in an inactive form. The active form binds to type-II TGF- $\beta$ receptor, which is activated to phosphorylate type-I TGF- $\beta$ receptor. Then the type-I protein and TGF- $\beta$ transcription-regulating complex translocates into the nucleus and exerts the effects of TGF- $\beta$ (26).

The major function of TGF- $\beta 1$ is the regulation of extracellular matrix deposition and fibrinogenesis. TGF- $\beta 1$ stimulates the proliferation of fibroblasts, induces production of collagen, proteoglycans, laminin and fibronectin, and inhibits the collagenase (27). TGF- $\beta 1$ inhibits fibrinolysis through the production of plasminogen and its conversion to plasmina. The factor also decreases the activity of the metalloproteinases and stimulates the production of protein receptors, which are known chemo-attractants for macrophages (23). All these activities lead to fibrosis and loss of renal function.

TGF- $\beta 1$ is excreted in the urine in normal conditions, in urological conditions such as vesicoureteral reflux and hydronephrosis, and in non-urological conditions $(6,28)$. In our control group, without renal pathology, the mean TGF- $\beta 1$ concentration was 35.8 $\pm 16.2 \mathrm{pg} / \mathrm{mL}$ SD. Palmer et al. reported a mean \pm SD of $26.6 \pm 6.3 \mathrm{pg} / \mathrm{mL}$ in their control group (9).

We found that bladder urine TGF- $\beta 1$ concentration in patients with obstructive hydronephrosis is almost 3-fold higher than that in controls without renal pathology. We also found that TGF- $\beta 1$ in renal pelvic urine is higher than in the bladder urine. These findings are in agreement with the data reported by Palmer et al. (9), Furness et al. (10), and El-Sherbiny et al. (28). Unlike other authors $(17,28)$, in our study group we did not include patients that presented or had a previous history of urinary infection, or those with vesicoureteral reflux. In the case of patients that required additional elements for post-pyeloplasty urinary drainage, such as pigtail catheters or nephrostomy tubes, we decided to measure TGF- $\beta 1$ four months after their removal. We believed that the presence of these elements could generate increased levels of TGF- $\beta 1$ due to the activation of pro-inflammatory factors and lead to a biased sample. 
With a TGF- $\beta 1$ cutoff point of $39.75 \mathrm{pg} / \mathrm{mL}$ in our study, we can conclude that patients with a TGF- $\beta 1$ level greater than 39.75 have a relative risk 4.25-fold higher of developing obstructive hydronephrosis.

Regarding the possible correlation between preoperative radiographic studies and urinary TGF$\beta 1$, we could determine that there is a correlation between the percentage of relative renal function loss on DMSA scintigraphy and the level of intraoperative TGF- $\beta 1$. However, no such correlation was found when we compared TGF- $\beta 1$ with preoperative bladder urine, presumably due to the dilution of TGF- $\beta 1$ by urine coming from the contralateral kidney.

We did not find a statistically significant relationship between renal scarring and urinary TGF- $\beta 1$. It is worth noting that six patients presented renal scars on the DMSA scan; however, their cause could not be identified. There was also no difference in urinary TGF- $\beta 1$ levels postoperatively between patients with or without renal scars. The presence of renal scars suggests fibrosis and collagen deposition in renal parenchyma. This is the sequelae of a process that may be absent at the time of measuring TGF- $\beta 1$ values. That is, renal scarring is not the cause of the elevated urinary TGF- $\beta 1$ concentration but the final consequence of this increase.

In their study of 35 children with ureteropelvic junction obstruction, Taha et al. found a significant increase in TGF- $\beta 1$ values at month one post pyeloplasty and a gradual decrease reaching a significantly low level one year postoperatively (29). In our study we observed that three and five months after surgery, bladder urinary TGF- $\beta 1$ decreased significantly to values closer to those of controls without renal pathology. In addition, there was a parallel decrease of almost $60 \%$ in hydronephrosis, compared to preoperative values. We believe a longer follow-up would not render different results on TGF- $\beta 1$ concentration that could contradict our findings reached at $3 / 5$ months. A further decrease in the renal pelvic diameter, however, may be expected over time.

These findings confirm that urinary TGF- $\beta 1$ is not only a useful tool for the diagnosis of upper urinary tract obstruction but also a reliable biomarker for the progression of the disease comparable to those traditionally used.
With respect to the chronological age of the patients, we could not find any significant correlation with the level of intrapelvic urine TGF- $\beta 1$ or the percentage of renal function loss in our study.

All the cases tested negative for microalbuminuria. The few cases in our study that had proteinuria and $\beta 2-\mathrm{MG}$ were normalized after pyeloplasty. No correlation could be found between proteinuria, $\beta 2-\mathrm{MG}$ and TGF- $\beta 1$, the grade of hydronephrosis or values on renal scintigraphy.

TGF- $\beta 1$ could therefore become a useful tool for the diagnosis of obstructive hydronephrosis as well as for the evaluation of the parenchymal function, pre- and postoperatively (29-32).

We currently have a group of 16 patients with non-obstructive hydronephrosis that are being followed-up and in which TGF- $\beta 1$ was measured (data not presented). We believe that in the case of this group, due to the erratic course of hydronephrosis, it is important to perform serial follow-up with ultrasound and evaluation of TGF- $\beta 1$ along the time, but these data will probably be material for further studies.

\section{CONCLUSION}

Urinary biomarkers may become potential tools for the diagnosis and follow-up of hydronephrosis. Currently, TGF- $\beta 1$ has shown to be useful in identifying hydronephrosis with early parenchymal injury and monitoring response to intervention. However, future and larger studies with longer follow-up time should be carried out in order to compare obstructive hydronephrosis with non-obstructive hydronephrosis as well as with controls to truly determine which patients would benefit from surgical repair.

\section{CONFLICT OF INTEREST}

None declared.

\section{REFERENCES}

1. Csaicsich D, Greenbaum LA, Aufricht C: Upper urinary tract: when is obstruction obstruction? Curr Opin Urol. 2004; 14: 213-7. 
2. Salem YH, Majd M, Rushton HG, Belman AB: Outcome analysis of pediatric pyeloplasty as a function of patient age, presentation and differential renal function. J Urol. 1995; 154: 1889-93.

3. Kass EJ, Majd M, Belman AB: Comparison of the diuretic renogram and the pressure perfusion study in children. J Urol. 1985; 134: 92-6.

4. Josephson S, Dhillon HK, Ransley PG: Post-natal management of antenatally detected, bilateral hydronephrosis. Urol Int. 1993; 51: 79-84.

5. Wen JG, Frøkiaer J, Jørgensen TM, Djurhuus JC: Obstructive nephropathy: an update of the experimental research. Urol Res. 1999; 27: 29-39.

6. Massagué J: The transforming growth factor-beta family. Annu Rev Cell Biol. 1990; 6: 597-641.

7. Chevalier RL: Growth factors and apoptosis in neonatal ureteral obstruction. J Am Soc Nephrol. 1996; 7: 1098105.

8. Kaneto H, Morrissey J, Klahr S: Increased expression of TGF-beta 1 mRNA in the obstructed kidney of rats with unilateral ureteral ligation. Kidney Int. 1993; 44: 313-21.

9. Palmer LS, Maizels M, Kaplan WE, Firlit CF, Cheng EY: Urine levels of transforming growth factor-beta 1 in children with ureteropelvic junction obstruction. Urology. 1997; 50: 769-73.

10. Furness PD 3rd, Maizels M, Han SW, Cohn RA, Cheng EY: Elevated bladder urine concentration of transforming growth factor-beta1 correlates with upper urinary tract obstruction in children. J Urol. 1999; 162: 1033-6.

11. Fernbach SK, Maizels M, Conway JJ: Ultrasound grading of hydronephrosis: introduction to the system used by the Society for Fetal Urology. Pediatr Radiol. 1993; 23: 478-80.

12. Schwartz GJ, Haycock GB, Spitzer A: Plasma creatinine and urea concentration in children: normal values for age and sex. J Pediatr. 1976; 88: 828-30.

13. Chambers RE, Bullock DG, Whicher JT: External quality assessment of total urinary protein estimation in the United Kingdom. Ann Clin Biochem. 1991; 28: 467-73.

14. Chambers RE, Bullock DG, Whicher JT: Urinary total protein estimation--fact or fiction? Nephron. 1989; 53: 33-6.

15. Evrin PE, Wibell L: The serum levels and urinary excretion of B2-Microglobulin in apparently healthy subjects. Scand J Clin Lab Invest. 1972; 29:69-74

16. Neil DR, Haycock GB: In: Holliday MA, Barrat TM, Avner ED (eds.), Laboratory Investigation. Pediatric
Nephrology. Buenos Aires, Lippincott Williams \& Wilkins. 1994; chapter 22: pp. 397-409.

17. Chiou YY, Chiu NT, Wang ST, Cheng HL, Tang MJ: Factors associated with the outcomes of children with unilateral ureteropelvic junction obstruction. J Urol. 2004; 171: 397-402; discussion 402.

18. Gulmi F, Felsen D, Vaughan E: Pathophysiology of Urinary Tract Obstruction. In: Walsh PC, Retik AT, Vaughan ED, Wein A (eds.), Campbell's Urology. Buenos Aires, Editorial Panamericana. 2004; vol. 1, pp. 450-2.

19. Massagué J: The TGF-beta family of growth and differentiation factors. Cell. 1987; 49: 437-8.

20. Toblli J: Sistema renina-angiotensina y daño tubulointersticial. Publicación de la Sociedad Argentina de Nefrología. 2005; 3: 47-52.

21. Nilsen-Hamilton M: Transforming growth factor-beta and its actions on cellular growth and differentiation. Curr Top Dev Biol. 1990; 24: 95-136.

22. Seremetis GM, Maizels M: TGF-beta mRNA expression in the renal pelvis after experimental and clinical ureteropelvic junction obstruction. J Urol. 1996; 156: 261-6.

23. Misseri R, Meldrum KK: Mediators of fibrosis and apoptosis in obstructive uropathies. Curr Urol Rep. 2005; 6: 140-5.

24. Murakami K, Takemura T, Hino S, Yoshioka K: Urinary transforming growth factor-beta in patients with glomerular diseases. Pediatr Nephrol. 1997; 11: 334-6.

25. Yang Y, Hou Y, Wang CL, Ji SJ: Renal expression of epidermal growth factor and transforming growth factor-beta1 in children with congenital hydronephrosis. Urology. 2006; 67: 817-21; discussion 821-2.

26. Schnaper HW, Hayashida T, Poncelet AC: It's a Smad world: regulation of TGF-beta signaling in the kidney. J Am Soc Nephrol. 2002; 13: 1126-8.

27. Postlethwaite AE, Keski-Oja J, Moses HL, Kang AH: Stimulation of the chemotactic migration of human fibroblasts by transforming growth factor beta. J Exp Med. 1987; 165: 251-6.

28. El-Sherbiny MT, Mousa OM, Shokeir AA, Ghoneim MA: Role of urinary transforming growth factor-beta1 concentration in the diagnosis of upper urinary tract obstruction in children. J Urol. 2002; 168: 1798800.

29. Taha MA, Shokeir AA, Osman HG, Abd El-Aziz AelA, Farahat SE: Pelvi-ureteric junction obstruction in children: the role of urinary transforming growth factor-beta and epidermal growth factor. BJU Int. 2007; 99: 899-903. 
30. Moses HL, Yang EY, Pietenpol JA: TGF-beta stimulation and inhibition of cell proliferation: new mechanistic insights. Cell. 1990; 63: 245-7.

31. Chevalier L, Roth J: In: Avner E, Harmon W, Niaudet (eds.), Obstructive Uropathy. Pediatric Nephrology.
Buenos Aires, Lippincott Williams \& Wilkins. 2004; chapter 55: pp. 1049-76.

32. Chevalier RL: Biomarkers of congenital obstructive nephropathy: past, present and future. J Urol. 2004; 172: 852-7.

Accepted after revision:

February 15, 2009

\author{
Correspondence address: \\ Dr. Cristian Sager \\ Pichincha 1880, C1245AAM \\ Buenos Aires, Argentina \\ Fax: + 5411 4308-0258 \\ E-mail: cristian.sager@gmail.com
}

\section{EDITORIAL COMMENT}

Hydronephrotic or obstructed, operation or observation, these are the two critical questions rising when a pediatric urologist is dealing with a hydronephrotic kidney. Unfortunately, today we still do not have the perfect tool to differentiate between obstruction and hydronephrosis, in order to decide when a hydronephrotic kidney is truly obstructed and should be operated. Moreover, we cannot forecast the functional prognosis of the operated unit in advance.

At present, we use ultrasound and isotope scan to assess a hydronephrotic kidney. Unfortunately, ultrasound cannot assess renal function, which is the most important factor for the decision whether to observe or operate. Isotope scans provides functional data about perfusion, concentration and excretion, however there is no standardization of these studies for children, inter exam deviations may occur due to change in the hydration and hemodynamic status of the child. Moreover, those studies expose the child to ionized radiation. Several other imaging modalities have been developed in order to improve our ability to diagnose accurately the child with renal obstruction, such as Computerized Tomography Fluoroscopy and quantitative Magnetic Resonance Urography. Both methods are still under investigation, and a standard protocol does not exist, CT is related to high dose of radiation and child anesthesia is needed especially for the MRI studies.

Biochemical and molecular markers may serve as the future markers for the assessment of hydronephrotic kidney. In general, I believe that biochemical and molecular changes in tissues under stress (such as obstruction, or malignancy) occur prior to functional and phenotypic changes, i.e. changes in the value of such marker will appear prior to tissue changes such as increased hydronephrosis and deterioration of function. The earned time gap will allow early decision about the proper management of that kidney.

Transforming growth factor beta-1 (TGF$\beta 1)$ was investigated as a potential marker for the diagnosis of obstruction. Several animal and human studies have already shown a direct relationship between increased level of TGF- $\beta 1$ and renal obstruction, significant difference in TGFß-1 levels were found between hydronephrotic non obstructed kidney obstructed kidneys and normal non obstructed non hydronephrotic kidney. The current study also demonstrated reduction in TGF- $\beta 1$ levels following the relief of the obstruction, maybe the decline slope 
of this factor and nadir level may also provide information about prognosis and the faith of the operated kidney.

The major obstacle in our ability to implement the use of TGF- $\beta 1$ for clinical decisions is the relatively small number of patients that have participated in these clinical studies. TGF- $\beta 1$ levels can be measured non-invasively in voided urine, technically it is relatively easy to collect even in children; a fact that should encourage further studies in order to clarify its potential in supporting clinical decisions in children with hydronephrosis and suspected renal obstruction.

\author{
Dr. Sarel Halachmi \\ Pediatric Urology Service \\ Bnai Zion Medical Center \\ The Faculty of Medicine \\ Technion Israeli Institute of Technology \\ Haifa, Israel \\ E-mail:Sarel.halachmi@b-zion.org.il
}

\section{EDITORIAL COMMENT}

The issue of non invasive diagnosis of ureteropelvic junction obstruction (UPJO) is an important topic that is of great interest to physicians and to the involved patients and their families. In this article investigators from Hospital de Pediatría Dr. J. P. Garrahan, Buenos Aires, Argentina did a prospective study to assess the role of transforming growth factor- $\beta 1$ (TGF- $\beta 1$ ) in congenital UPJO at diagnosis and during postoperative follow-up.

Although the results of the current study are intriguing, the rationale for using TGF- $\beta 1$ for diagno- sis of UPJO is not clear. Several factors are important to consider when evaluating the data in this article. The small number of cases is one of the shortcomings. Furthermore, the effect of some variables like patient age, degree of hydronephrosis was not evaluated. Overall, this article adds a little data to the previous publications in this area. The conclusions described should be confirmed by another well done prospective study on a larger number of cases.

Dr. Osama M. Sarhan Urology and Nephrology Center Faculty of Medicine, Mansoura University Mansoura 35516, Egypt E-mail:o_sarhan2004@yahoo.com

\section{EDITORIAL COMMENT}

The purpose of this study was to determine the usefulness of measuring transforming growth factor- $\beta 1$ (TGF- $\beta 1$ ) as well as several other urinary markers in children with ureteropelvic junction (UPJ) obstruction as compared to normal controls. In addition, the authors used TGF- $\beta 1$ as a post-operative marker to correlate radiographic success after pyelo- plasty. Several similar studies have been previously published in the literature, as cited in References 9-10, 25, 28-29.

This study examined a relatively small number of study patients (19) with presumed UPJ obstruction and the follow-up period was fairly short (3 - 5 months after surgery, depending on whether 
post-operative drains were necessary). Longer follow-up might have shown even more dramatic results with both TGF- $\beta 1$ levels and renal pelvic diameter as the authors allude to in the Discussion section.

Of the 19 renal scans performed, 11 had either scars, reduced differential function in the affected kidney $(<40 \%)$ or both. Eight patients $(42 \%)$ did not have reduced function or scars and the diagnosis of UPJ obstruction was made on a single DTPA renal scan washout curve, even though there was preserved renal function on DMSA scan. There was no statistical correlation found between urinary TGF- $\beta 1$ levels and renal scarring on DMSA scan.

In order to make their case for TGF- $\beta 1$ stronger, the authors could have also compared levels of patients with hydronephrosis and an unobstructed washout curve to the present study and control groups. With all of the fallacies of diuretic renal scan, discriminating between hydronephrosis with and without obstruction is much more useful than between their study group and normal controls. The authors mention at the end of the Discussion that they have a group of 16 patients with this non-obstructive hydronephrosis but they chose not to present this data at this time. If TGF- $ß 1$ levels in these patients were found to be statistically closer to normal levels, this data may prove the true usefulness of a noninvasive urinary marker such as TGF- $\beta 1$ to predict who would benefit from early surgical intervention to prevent renal functional loss if it could discriminate between obstructive and non-obstructive hydronephrosis with accurate sensitivity and specificity.

The authors' data seem to agree with prior studies that TGF- $\beta 1$ levels are higher in the urine of those with obstruction as compared to controls and this number decreases to a value closer to that of normal controls at least 3-5 months after pyeloplasty. It remains to be seen whether their conclusions regarding TGF- $\beta 1$ will come to fruition as a useful biomarker to identify early parenchymal injury in obstructive uropathy. Clearly, this is a very desirable goal and several small studies have suggested that this may be the case, but until larger studies with longerterm follow-up can be completed, the usefulness of urinary TGF- $\beta 1$ as a biomarker for everyday clinical practice is still unclear. I agree with the authors' final statement that future studies comparing TGF- $\beta 1$ in obstructive hydronephrosis versus non-obstructive hydronephrosis and controls may help to determine which patients might benefit from early surgical repair to prevent renal parenchymal loss.

Dr. Seth A. Alpert Clinical Assistant Professor Section of Pediatric Urology Nationwide Children's Hospital Columbus, Ohio, USA E-mail: seth.alpert@nationwidechildrens.org 